\title{
Influencia de la cultura académica de origen en el desempeño docente de profesores universitarios noveles
}

\author{
Juan Patricio SÁNCHEZ-CLAROS \\ Universidad de Málaga \\ jpsc@uma.es
}

\begin{abstract}
Resumen:
En esta investigación se estudia la influencia de la cultura académica de origen en las creencias y la práctica docente de dos profesores universitarios noveles procedentes de distintas disciplinas, mediante sendas entrevistas. Entre las conclusiones alcanzadas, pueden mencionarse: la cultura académica es considerada como un constructo emergente, explicitado a través de una visión didáctica; la influencia es tanto menos conflictiva cuanta mayor es la continuidad disciplinar y la integración en la estructura departamental y simbólico-cultural
\end{abstract}

Palabras clave: EEES; desempeño docente; cultura académica; profesorado novel

\section{Influence of the academic culture in the teacher performance of novice university teachers}

\begin{abstract}
:
This paper discusses the influence of the academic culture of origin in beliefs and teaching practice of two novice academics from different disciplines, using individual interviews. Among the conclusions reached may be mentioned: academic culture is seen as an emerging construct, explicited through a didactical vision; the influence is much less confrontational how much greater the disciplinar continuity and the integration in the departmental and symbolic-cultural structure.
\end{abstract}

Key Words: EHEA; teacher performance; academic culture; novice teachers

\section{Referencia normalizada:}

Sánchez Claros, J. P. (2014): Influencia de la cultura académica de origen en el desempeño docente de profesores universitarios noveles. Historia y Comunicación Social. Vol. 19. Núm. Especial Febrero. Págs. 523-533.

Sumario: 1. Introducción. 2. Desempeño docente. 2.1. La construcción de la identidad docente. 2.2. Las creencias del profesorado novel. 3. Práctica docente. 3.1. Aproximación a los métodos en el EEES. 4. Cultura académica. 4.1. La inmersión en una cultura académica. 4.2. Socialización previa del docente novel. 5. Diseño. 6. Análisis. 6.1. Actitud psicológica frente a la ejecución en el aula. 6.2. Respuesta efectiva ante la percepción inicial. 6.3. Manifestación de la socialización académica previa como extracción de recursos. 6.4. Carácter atribuido a la cultura académica de origen. 6.5. Sentido de adscripción a la institución: eventual carácter de continuidad. 6.6. Organización curricular. 6.7. Decisiones metodológicas. 7. Conclusiones. 8. Bibliografía. 


\section{Introducción}

La implantación paulatina del Proceso de Bolonia, junto con el objetivo de homogeneizar las titulaciones e incrementar la presencia y la importancia de las universidades europeas a nivel mundial, ha implicado también la introducción de una serie de modificaciones didácticas que han afectado a las prácticas docentes y a la actitud del alumnado frente a su aprendizaje. El énfasis puesto en la diversificación metodológica, en la incentivación de la investigación, en el fomento del trabajo en grupos o por proyectos, y en el desarrollo de la autonomía de los alumnos como protagonistas de la relación pedagógica, ha venido a subvertir en ocasiones algunos hábitos que gozaban de tradicional asentamiento entre las prácticas docentes que se venían llevando a cabo. La renovación de la función docente y una actitud del profesorado más como facilitador del aprendizaje que como transmisor del conocimiento son los aspectos fundamentales que han afectado a las prácticas docentes.

Sin embargo, entre todos los colectivos docentes, el del profesorado novel se revela como uno de los afectados de manera más intensa, viéndose enfrentados a un doble condicionante en su desempeño: por un lado, el correspondiente a la presión de integrarse en equipos y departamentos que cuentan ya con sus propias dinámicas de funcionamiento; y por otro, la atmósfera que en esos mismos departamentos ha creado la implantación de las novedades del Espacio Europeo de Educación Superior. Estas novedades han supuesto diferentes rutas de acomodación, en virtud de los diferentes usos didácticos y metodológicos a que tradicionalmente dan lugar las distintas disciplinas académicas. Disciplinas académicas en las que se ha forjado precisamente la socialización previa de estos profesores noveles.

\section{Desempeño docente}

\subsection{La construcción de la identidad docente}

Con la expresión "desempeño docente" entendemos el conjunto de la actividad profesional del profesor universitario. Se encuentra constituida por las acciones concretas de carácter educativo llevadas a cabo en el aula, que incluyen la planificación y la selección de material. Pero también lo constituye el conjunto de creencias, actitudes y expectativas del docente en torno a la enseñanza y a su propio papel como profesional. Este conjunto de elementos se va conformando durante la etapa de socialización previa del profesorado dentro de la disciplina epistemológica a la que pertenecen, durante su formación como estudiantes así como durante su integración en el departamento al que se encuentran adscritos, cuya cultura y usos van pasando a formar parte de la identidad docente en formación, de tal manera que se van atendiendo como un conjunto de normas a las que hay que atenerse (Gewerc, 1996). 
De esta forma, la identidad es una mezcla de vivencias personales, cultura, influencia del medio y valores institucionales, que pueden moldearse según la función cumplida, las circunstancias y especialmente, el paso del tiempo de permanencia en la institución (Day, 2000). Dada la frágil posición inicial de los noveles, esta adscripción se lleva a cabo mediante un amoldamiento a las expectativas del entorno, de acuerdo a la idea expresada por Lacey (1977) de "conformidad estratégica", un paso previo en la construcción de la identidad docente propia.

\subsection{Las creencias del profesorado novel}

El profesor universitario extrae sus creencias a partir de su experiencia educativa, integrándola en sus modos de actuar y de pensar y que se muestran prácticamente en el desempeño docente de aula (Marín Díaz, 2004; Escartín, 2008). Joram y Gabrielle (1998) detallan esta visibilización de la identidad docente señalando caracteres y situaciones, como la institución de pertenencia, la comunidad científica, las relaciones con el alumnado, la experiencia adquirida en docencia e investigación, la formación continuada, la asistencia a jornadas, cursos, talleres u otros eventos, etc. Todo lo cual abunda en la idea de que las creencias representan un elemento de constitución identitario que necesariamente ha de manifestarse a través de una vertiente práctica y dinámica.

Suponen asimismo un factor muy importante para los noveles pues determinan la construcción de teorías que sustentan su ejecución práctica y sus modos de relación con el entorno (Herber y Worthy, 2001), teorías que precisan de un proceso de reflexión sobre la práctica (Prieto Navarro, 2007) para determinar su integración como elementos de identidad. Ello implica, como indica Pérez Gömez (1988), movilizar el mundo de la experiencia, con sus valores, símbolos, connotaciones, intereses y escenarios determinados social y políticamente.

Un rasgo determinante en este tránsito es el referido a la preocupación por el contenido de la materia impartida, pues se entiende que tal conocimiento refleja no sólo el dominio de la disciplina, sino el modo óptimo de acercamiento a la calidad profesional (Gros y Romañá, 2004). No obstante, una visión menos tradicional vería al profesor como un compañero de los estudiantes que se une a ellos en la búsqueda de un mejor entendimiento de la disciplina (Bain, 2007).

\section{Práctica docente}

\subsection{Aproximación a los métodos en el EEES}

Todos estos elementos se hacen visibles, como ya se ha mencionado, a través de las prácticas concretas. Entre ellas, cobran especial singularidad los procesos de selección de métodos y modalidades organizativas, en tanto que elementos fundamentales de la 
práctica docente y del ejercicio profesional (Zabalza, 2003). Tal proceso de selección es uno de los rasgos que evidencian mayor dependencia de la cultura académica de la disciplina que se imparte. El Espacio Europeo de Educación Superior ha enfatizado el paso desde una educación históricamente fundamentada en la enseñanza dictada por el profesor, hacia un modelo en el que el alumno cobre protagonismo como sujeto activo de su propio aprendizaje, y en el que el profesor es un facilitador en esta vía hacia el conocimiento. Otra consecuencia es la necesidad de diversificación metodológica y de modalidades organizativas, con las que atender a estas nuevas exigencias.

Esta diversificación, que procura superar el antiguo monopolio exclusivo de la lección magistral, incluye técnicas de Aprendizaje Basado en Problemas (ABP) (García Sevilla, 2008; Escribano y Del Valle, 2010; Araújo y Sastre, 2008; Sánchez González. 2010), supuestos prácticos dramatizados o role-playings (Sánchez González, 2010), o el uso de mapas conceptuales (González García, 2000). En cuanto a la organización, la tutoría académica (Gairín et al., 2004; García Nieto et al., 2005; Paredes y De La Herrán, 2010; Rodríguez Espinar, 2004) y la carpeta o portafolio docente (Cano, 2005; De Rijdt et al., 2006) se presentan como formas recomendadas de seguimiento académico del alumnado.

\section{Cultura académica}

\subsection{La inmersión en una cultura académica}

De acuerdo con Austin (1992), podemos distinguir tres tipos de culturas en las que los profesores universitarios se socializan a lo largo de su vida profesional:

- Cultura disciplinar. Supone que cada disciplina tiene sus propias tradiciones, formas de pensamiento, métodos de investigación, orientación hacia la enseñanza, tópicos, símbolos y referencias bibliográficas que determinan el campo de estudio y las relaciones internas.

- Cultura de la profesión académica. De este modo, las distintas facultades y escuelas universitarias presentan diferentes funciones, estructura organizativa, objetivos, creencias y concepciones de la realidad, que se manifiestan en la forma de organizar el trabajo o en la interacción con los alumnos.

- Cultura institucional. Marca las reglas internas de poder y establece las situaciones normalizadas de ejercicio profesional y de desarrollo de hábitos.

Por su parte, centrándose en el proceso de enseñanza-aprendizaje, López Noguero (2005) distingue entre un modelo tradicional, centrado en el profesor que es quien señala los objetivos, dirige la clase y evalúa contenidos adquiridos; y un modelo participativo, en el que se valoran los aprendizajes procedimentales, el trabajo en equipo, la comunicación y la creatividad. A pesar de estas notorias diferencias, en la 
práctica ambos modelos han de ser considerados como instrumentos al servicio de los objetivos de aprendizaje, y no como fines en sí mismos.

\subsection{Socialización previa del docente novel}

La socialización académica previa de los profesores universitarios noveles, ya desde su etapa de estudiantes, se enmarca dentro de la noción de "conocimiento tácito" (Geertz, 1994: Gerholm, 1990) por la que se ponen en funcionamiento los rituales de socialización. Éstos contienen sus propias redes simbólicas y ambiente epistémico que forman el conocimiento práctico del aprendiz, y que es indisoluble tanto de la estructura organizacional en la que se encuentra (Pérez Gómez, 2010) como de los diferentes usos institucionales que configuran la práctica profesional (Gimeno, 1988). En definitiva, este proceso socializador se asienta sobre un conjunto de rutinas establecidas (Rumbo Arcas, 1998), basadas en aquello que vivieron con los profesores que les precedieron (López Noguero, 2005; Manso Martínez, 2009) y que termina por construir una imagen heredada de lo que es ser profesor (Prieto, 2008). Se trata por tanto de un rol aprendido que tiende a reproducir estrategias consideradas válidas o exitosas.

\section{Diseño}

En esta investigación se confrontan las visiones que tienen sobre su actividad docente dos profesores universitarios noveles con experiencias de socialización académica diferenciadas. Fueron seleccionados según un muestreo criterial de entre los pertenecientes al Seminario de Formación Docente para el Profesorado Universitario $2^{\mathrm{a}}$ Fase, implantado en la Universidad de Málaga. Se procuró que ambos sujetos perteneciesen a culturas disciplinarias diferenciadas. Se determinó asimismo que el carácter de "novel" hiciese referencia a una experiencia docente no superior a dos años. El primer seleccionado es perteneciente a una departamento de la Escuela Técnica Superior de Ingeniería Informática, quien accede a la docencia desde una beca de investigación que le ha propiciado un estrecho contacto con el departamento. El segundo sujeto es una profesora de la Facultad de Ciencias de la Educación, con una titulación de Psicopedagogía, y cuya docencia procede de una bolsa de trabajo para Profesores Sustitutos Interinos, teniendo en consecuencia una procedencia externa a la institución universitaria.

Para realizar el estudio de casos, se han empleado sendas entrevistas semiestructuradas en torno a categorías extraídas de la revisión teórica. El modelo de análisis llevado a cabo presenta un carácter mixto que reúne los modelos de interpretación de significado y de lectura teórica (Kvale, 2011), priorizando el elemento narrativo que respeta el sentido del relato, en torno a una serie de categorías que se explicitan en el siguiente análisis. 


\section{Análisis}

\subsection{Actitud psicológica frente a la ejecución en el aula}

El rasgo principal asociado a la ejecución en el aula de estos docentes es el relativo al miedo escénico, desde el momento en que el inicio de la clase se identifica con la realización de una suerte de performance que requiere tanto la atención de la audiencia como la obtención de su participación en las actividades. Para ambos componentes, el dominio de los recursos y la experiencia previa se consideran las herramientas necesarias.

Así, mientras que el primer sujeto valora su experiencia docente impartiendo cursos en Diputación, la profesora PSI relaciona el miedo escénico con su carencia de recursos previos. Ambos indican el elevado número de alumnos como una dificultad, pero mientras que el primero lo asume como parte de la responsabilidad que le corresponde, la segunda lo ve como una preocupación añadida, a lo que añade la edad de parte de su alumnado, constituida por maestros en ejercicio.

\subsection{Respuesta efectiva ante la percepción inicial}

El reconocimiento de la propia capacidad de respuesta es el siguiente factor, que puede incidir tanto positiva como negativamente, si se entiende que los recursos disponibles son insuficientes. Para el primer sujeto, su experiencia previa, la asistencia de su tutor y su integración en el departamento operan como elementos de confianza con los que dar respuesta a los problemas. La cuestión fundamental es la continuidad entre la autoestima a que da lugar esta confianza, y el desempeño mismo, que se ve así facilitado. En cambio, la segunda profesora, que no cuenta con estos factores de confianza, ve las dificultades como retos a superar, y esta consideración constituye su estrategia de afrontamiento para evitar que los problemas paralicen su ejecución.

6.3 Manifestación de la socialización académica previa como extracción de recursos

En ambos profesores, la reflexión sobre la formación docente, los modos de llevar a cabo la práctica, el diseño del currículum o las decisiones metodológicas son el fundamento de sus preocupaciones didácticas. De hecho, las culturas respectivas de socialización académica se entendían como almacenes de recursos didácticos de donde extraer el instrumental profesional del docente. Esto incluía tanto recursos materiales como personales, en la forma de conversaciones, sugerencias o consejos bien de los tutores o de otros compañeros.

Esta extracción de recursos como elemento fundamental es extendido por ambos sujetos a los supuestos de cursos y congresos por el primer profesor, o a cursos y oposiciones por parte de las segunda, lo que supone una extensión de la experiencia previa fuera de los límites estrictos de la escolaridad o del mundo universitario. 


\subsection{Carácter atribuido a la cultura académica de origen}

Ambos sujetos integran a la cultura universitaria como un eje de continuidad en su desempeño, pero las diferencias provienen al considerar sus distintos modos de acceso a la función docente. Para el primer sujeto, la regla es la de la continuidad. Participa con su tutor en la impartición de la asignatura, y su visión de la cultura de origen no es conflictiva. Frecuentemente menciona la necesidad de la adscripción a un "método" -en consonancia con el carácter de ciencia "pura" de la disciplina a la que pertenece- cuyo seguimiento didáctico va referido en términos de utilidad, bien sea como mecanismo propedéutico para la comprensión de los estadios más avanzados de la asignatura, o bien como facilitador del trabajo de los alumnos, que se ven así libres de cualquier agregado crítico respecto de un material que se les proporciona bajo la forma de datos empíricos.

El segundo sujeto, al no provenir exactamente del mismo campo académico en el que imparte docencia, ha necesitado una aproximación analógica entre disciplinas basado en la idea del aire de familia entre campos epistemológicos vecinos que de algún modo rompe con la idea de continuidad que caracterizaba a su colega. Para ella, la búsqueda de una identificación cultural y la obtención de recursos específicos se hace más necesaria y perentoria.

\subsection{Sentido de adscripción a la institución eventual carácter de cotinuidad}

La socialización académica está inserta dentro de un contexto institucional que la envuelve y donde los sujetos se desarrollan. Para el primer profesor, su adscripción proviene de una relación becaria que le he permitido una integración progresiva y una igualmente progresiva forja identitaria. Su desarrollo profesional se adecua a la estructura de adscripción administrativamente preestablecida, cuyos presupuestos y pasos no son puestos en cuestión. Se presenta así como una persona integrada en el sistema, que acepta las reglas de juego de la institución y el camino de desarrollo profesional que tal integración le ofrece.

En contraste, la vinculación a la institución de la segunda profesora es más débil. En primer lugar se encuentra la falta de adecuación inmediata entre su disciplina de origen y aquella en la que trabaja. Su adscripción departamental es menos sólida que en su compañero, y las perspectivas de continuidad profesional en el futuro, menos evidentes. Así, a la fragilidad propia del profesor universitario novel, debe añadir una serie de fragilidades añadidas para cuyo afrontamiento ha adoptado la perspectiva, ya mencionada, de reto que superar, una noción que aparece con gran frecuencia en su discurso.

\subsection{Organización curricular}

En cuanto a esta categoría, ambos señalan por igual la rigidez de la asignación docente, y la idea de que la programación condiciona y determina la actividad a desarrolla. El primer profesor apunta al hecho de ceñirse al método, la enseñanza del 
método que es prioritaria en su disciplina, y que impregna todas las decisiones curriculares. La segunda apunta al seguimiento de la programación, ciñéndose a lo que ésta determine, también como un requisito explícito procedente del departamento. En ambos casos, la programación y la organización curricular es un constructo normativo impuesto sobre el docente que regula su actuación y sobre el que apenas pueden realizarse retoques.

\subsection{Decisiones metodológicas}

En esta categoría la divergencia principal entre ambos se refiere a la consideración que otorgan a la innovación como factor de cambio de los usos didácticos recibidos. Mientras el primer profesor alude a la utilidad de la enseñanza del método de la manera heredada, por los motivos expuestos (valor propedéutico, beneficio para el alumno, etc.), la segunda opta por valorar el pensamiento crítico y autónomo de los alumnos. Se muestra así una de las más claras diferencias generalmente señaladas entre disciplinas. La función de la innovación, para el primer profesor, no debe afectar al núcleo epistemológico de la disciplina, sino más bien actuar de manera periférica como un incentivo de carácter motivador para la atención y la implicación de los alumnos, y siempre en la fase de aplicación práctica de lo previamente expuesto de forma teórica. La segunda profesora es mucho más flexible y crítica, entendiendo la innovación no como un factor externo de motivación, sino como un elemento intrínseco a la relación de enseñanza-aprendizaje.

\section{Conclusiones}

Como síntesis, las ideas fundamentales que pueden extraerse de los apartados previos, pueden resumirse en los siguientes puntos:

- El desempeño docente de los profesores universitarios noveles estudiados se encuentra determinado por la socialización académica previa en sus respectivas culturas académicas de origen.

- Éstas son consideradas como constructo emergente, que se manifiestan a través de una visión "didáctica", esto es, como el conjunto de decisiones didácticas que los sujetos reproducen, o ponen en cuestión.

- Tales culturas tienen efectos en la flexibilidad de uso de las metodologías, en consonancia con el perfil rígido o crítico de las distintas disciplinas.

- La influencia de su etapa como alumnos resulta tanto más relevante cuanto más próxima en el tiempo es a su incorporación a la función docente.

- Son relacionadas fundamentalmente con los métodos didácticos empleados. 
- El contenido de la cultura profesional se extiende a facetas de experiencia vital y de aprendizaje docente extrauniversitarios, abarcando ámbitos de pedagogía no formal.

- La influencia es tanto menos conflictiva, cuanta mayor es la continuidad disciplinar y la integración en la estructura departamental y simbólico-cultural.

- El desempeño práctico y los problemas de organización de aula son preocupaciones prioritarios.

- La categoría emergente más significativa surgida se refiere a la diversidad de modos de acceso a los cuerpos docentes universitarios, que implican diferentes tipos de relación con la estructura organizacional y departamental de la institución, el proceso de socialización y la disponibilidad de recursos. Se ha revelado de tal importancia que viene a explicar la mayoría de las más relevantes diferencias entre ambos sujetos, especialmente en la percepción subjetiva de su situación respecto de la función que cumplen y sus circunstancias, así como en la asunción de continuidad en el proceso de integración en la función docente universitaria. La adscripción continua es la generalmente considerada. Sin embargo, la consideración de los distintos modos de acceso podría arrojar luz de cara a la comprensión de situaciones específicas, incluyendo las vías que priman la procedencia "externa" a la institución universitaria.

\section{Bibliografía}

ARAÚJO, Ulisses y SASTRE, G.a (coords.) (2008). El aprendizaje basado en problemas: una nueva perspectiva en la enseñanza en la Universidad. Barcelona: Gedisa.

AUSTIN, A. (1992). "Faculty Cultures". En CLARK, B. y NEAVE, G. (2001). The Encyclopedia of Higher Education 3, Analytical Perspectives. Oxford; Pergamon Press.

BAIN, K. (2007). Lo que hacen los mejores profesores de universidad. Valencia: Universitat de València.

CANO, E. (2005). El portafolios del profesorado universitario. Un instrumento para la evaluación y para el desarrollo profesional. Barcelona: Octaedro.

DAY, C. (2006). Pasión por enseñar: la identidad personal y profesional del docente y sus valores. Madrid: Narcea.

DE RIJDT, C. (et al.) (2006). "Teaching portfolios in higher education and their effects: An explorative study". En: Teaching and Teacher Education, 22, p. 10841093.

ESCARTÍN SOLANELLES, J. (et al.) (2008). El docente novel, aprendiendo a enseñar. Barcelona: Octaedro. 
ESCRIBANO, A. y DEL VALLE, A. (cords.) (2010). El aprendizaje basado en problemas (ABP): una propuesta metodológica en Educación Superior. Madrid: Narcea.

GAIRÍN, J. (et al.) (2004). "La tutoría académica en el escenario europeo de la Educación Superior". En: Revista Interuniversitaria de Formación del Profesorado, 18 (1), p. 61-77.

GARCÍA SEVILLA, J. (coord.) (2008)- El aprendizaje basado en problemas en la enseñanza universitaria. Murcia: Universidad de Murcia.

GEERTZ, C. (1994). Conocimiento local: ensayos sobre la interpretación de las culturas. Barcelona: Paidós.

GERHOLM, T. (1990). "On tacit knowledge in Academia". En: European Journal of Education 25, 3. p. 263-271.

GEWERC, A. (1996). "Profesores universitarios: contextos organizativos y desarrollo profesional". En: Enseñanza, 14. p. 65-79.

GIMENO SACRISTÁN, J. (1988). El currículum: una reflexión sobre la práctica. Madrid: Morata.

GONZÁLEZ GARCÍA, F. (et al.) (2000). Una aportación a la mejora de la calidad de la docencia universitaria: los mapas conceptuales. Pamplona: Universidad Pública de Navarra.

GROS, B. y ROMAÑÁ, T. (2004). Ser profesor: palabras sobre la docencia universitaria. Barcelona: Octaedro.

HERBERT, E. y WORTHY, T. (2001). "Does the first year of teaching have to be a bad one? A case study of success". Teaching and Teacher Education, 17 (8), 879-911.

JORAM, E. y GABRIELLE, A. J. (1998). "Pre-service teacher's prior beliefs: transforming obstacles into opportunities". En: Teaching and Teacher Education, 14 (2), p. 175-191

KVALE, S. (2011). Las entrevistas en investigación cualitativa. Madrid: Morata.

LACEY, C. (1977). The socialization or teachers. Suffolk: Methuen.

LÓPEZ NOGUERO, F. (2005). Metodología participativa en la enseñanza universitaria. Madrid: Narcea.

MANSO MARTÍNEZ, J. M. (1999). "Profesionalización pedagógica del profesorado universitario". En: Revista Interuniversitaria de Formación del Profesorado, 34, p. 319-328.

MARÍN DÍAZ, V. (2004) Las creencias del profesor universitario en el siglo XXI. Córdoba: Universidad de Córdoba.

PAREDES, J. y DE LA HERRÁN, A. (coords.) (2010). Cómo enseñar en el aula universitaria. Madrid: Pirámide.

PÉREZ GÓMEZ, A. I. (1988). "El pensamiento práctico del profesor: implicaciones en la formación del profesorado". En VILLA, A. (coord.) (1988). Perspectivas y problemas de la función docente. Madrid: Narcea.

(2010). "La naturaleza del conocimiento práctico y sus implicaciones en la formación de docentes". En: Infancia y Aprendizaje, 33 (2), p. 171-177. 
PRIETO NAVARRO, L. (2007). Autoeficacia del profesor universitario. Eficacia percibida y práctica docente. Madrid: Narcea.

(2008). La enseñanza universitaria centrada en el aprendizaje. Estrategias útiles para el profesorado. Barcelona: Octaedro.

RODRÍGUEZ ESPINAR, S. (coord.) (2004). Manual de tutoría universitaria. Recursos para la acción. Barcelona: Octaedro.

RUMBO ARCAS, B. (1998). La calidad de la enseñanza universitaria y el desarrollo profesional de su profesorado. Granada: Grupo Editorial Universitario.

SÁNCHEZ GONZÁLEZ, M. P. (coord.) (2010) Técnicas docentes y sistemas de evaluación en educación superior. Madrid: Narcea.

ZABALZA, M. A. (2003). Competencias docentes del profesorado universitario: calidad y desarrollo profesional. Madrid: Narcea.

\section{El autor}

Juan Patricio Sánchez-Claros es profesor en el Departamento de Didáctica de la Música y las Artes Plásticas de la Facultad de Educación de la Universidad de Málaga, y docente en el Centro de Formación del Centro Municipal de Informática del Ayuntamiento de Málaga. Diplomado en Magisterio y Licenciado en Pedagogía por la Universidad de Málaga. Máster en Políticas y Prácticas de Innovación Educativa, y Máster en Intervención Social y Comunitaria por la Universidad de Málaga. Doctorando en el Departamento de Didáctica y Organización Escolar de la Facultad de Ciencias de la Educación de la Universidad de Málaga. Desarrollando la investigación de Tesis bajo la dirección de Ángel I. Pérez Gómez, sobre Educación Superior, Cultura Universitaria y Profesorado Novel Universitario. 